\title{
Transmodernidad: dos proyectos disímiles bajo un mismo concepto
}

\author{
Aldo Ahumada Infante \\ Universidad Academia de Humanismo Cristiano, Santiago, Chile. \\ Email: aahumadainfante@gmail.com
}

\begin{abstract}
Resumen: El presente trabajo aborda el concepto de Transmodernidad bajo dos puntos de vista diferentes. Por un lado, la mirada de Rosa María Rodríguez Magda, la cual nos plantea la Transmodernidad como una tríada dialéctica entre Modernidad-Postmodernidad-Transmodernidad, siendo esta última tanto una continuación como una superación de la Postmodernidad. El otro punto de vista corresponde a Enrique Dussel, el cual nos plantea la Transmodernidad como un proyecto utópico que es transversal a la Modernidad/Postmodernidad; está antes de la Modernidad y a la vez la trasciende, siendo una categoría de exterioridad que pretende sacar la voz propia de las culturas no europeo-norteamericanas, para así poder establecer diálogos interculturales simétricos que llevan consigo características de la propia cultura inconmensurables al proyecto moderno europeo. Así enfrentamos ambas concepciones de la Transmodernidad para destacar sus diferencias y contraposiciones.
\end{abstract}

Palabras clave: Transmodernidad, Modernidad, Postmodernidad, Postcolonialismo.

\section{Transmodernity: two dissimilar projects under the same concept}

\begin{abstract}
The following paper addresses the concept of Transmodernity under two different points of view. On one hand, the view of Rosa María Rodríguez Magda, which portrays the concept of Transmodernity as a dialectical triad between Modernity, Postmodernity and Transmodernity, being the last both continuation and overcoming of Postmodernism. On the other hand, the view of Enrique Dussel, who establishes Transmodernity as a utopian project that is transverse to modernity/postmodernity. This project is before modernity and transcends it as well, being a category of externality that seeks to raise the voice of non-European-American cultures, in order to establish symmetrical intercultural dialogues that carry features of their own culture immeasurable to the modern European project. This is how we'll confront both concepts of Transmodernity in order to highlight its differences and contrapositions.
\end{abstract} colonialism.

Key words: Transmodernity, Modernity, Postmodernity, Post-

\section{Transmodernidade: dois projetos diferentes sob o mesmo conceito}

Resumo: Este artigo aborda o conceito de transmodernidade sob dois pontos 
de vista diferentes. Por um lado, o olhar de Rosa María Rodríguez Magda, que se apresenta a transmodernidade como uma tríade dialética entre modernidade-pósmodernidade-transmodernidade, sendo este último tanto uma continuação e melhoria do pós-modernismo. O outro ponto de vista corresponde a Enrique Dussel, o que levanta a transmodernidade como um projeto utópico que é transversal ao modernismo / pós-modernismo, isto é antes de modernidade e ainda a transcende sendo uma categoria de externalidade que visa tornar o própria voz das culturas não-europeu-americanos, a fim de estabelecer diálogos interculturais simétricos para transportar as características imensuráveis da própria cultura para projecto europeu moderno. Confronta-se ambos conceitos de transmodernidade para destacar suas diferenças e conflitos.

Palavras-chave: transmodernidade, Modernidade, Pós-modernidade, Enrique Dussel, Magda Rodriguez , Postcolonialism.

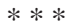

\section{Introducción}

El presente trabajo analiza el concepto Transmodernidad bajo dos puntos de vista diferentes, los cuales en cierta medida se presentan ideológicamente como antagónicos: hablamos de las concepciones de Rosa María Rodríguez Magda y de Enrique Dussel. La visión de la primera plantea el concepto transmoderno como una superación de la postmodernidad, como una radiografía del estado de la cultura en que vivimos hoy en día. Así la Transmodernidad es en cierta forma una continuación de la postmodernidad, una prolongación de ella; la trasciende. Enrique Dussel en tanto, ve la Transmodernidad como proyecto que parte desde más allá de la modernidad, atravesándola de modo transversal; es una irrupción desde las culturas no pertenecientes a la europeo-norteamericana, las cuales asumen los desafíos de la modernidad, e inclusive de la postmodernidad, pero que responden desde otro lugar, desde el lugar de sus experiencias particulares, es decir, desde una "exterioridad”. Con esto queremos indicar que el lugar del discurso de Rodríguez Magda se encuentra dentro de lo que Dussel llamaría “el centro” a diferencia de este último que plantea un hablar desde fuera de la totalidad, fuera de la modernidad como proyecto de occidente totalizante, en otras palabras es un hablar desde la exclusión.

Para poder analizar las posiciones de estos autores, tendremos que hablar de la modernidad bajos ambas ópticas, por un lado tenemos la tríada modernidad-postmodernidad-transmodernidad, analizada por Rodríguez Magda; ella nos recalca que esta última etapa, la Transmodernidad, es el momento actual en que vivimos como sociedad, en donde encontramos la emergencia de la virtualidad, la era de la información y globalización, además de un recoger la herencia de los retos abiertos de la modernidad que no sucumbieron a la postmodernidad, como se analizará más adelante. Es el proyecto transmoderno como discurso antihegemónico de la exterioridad de occidente, proyecto paralelo a la modernidad que intenta establecer diálogos interculturales simétricos. Transmodernidad como alternativa a la modernidad y a la postmodernidad. Por lo tanto, es necesario entender el 
proyecto moderno desde su definición intra-europea, en primer lugar, y por otro, la modernidad analizada desde la exterioridad, en la que se afirma que no hay modernidad sin “colonialidad,” centrando su análisis desde los parámetros del sistema-mundo (Wallerstein), sin remitirse a una Europa ensimismada.

\section{Desarrollo}

\section{a. La Modernidad desde Rosa María Rodríguez Magda}

Según Rodríguez Magda, y tomando a Habermas como referencia, el proyecto de la Modernidad es el esfuerzo ilustrado por desarrollar desde la razón las esferas de la ciencia, la moralidad y el arte, separadas de la metafísica y la religión (2007); además de ello:

"Representa una mirada puesta en el futuro; es en él, y no en la imitación del pasado, donde el individuo piensa encontrar la realización de sus expectativas más o menos utópicas; lo nuevo atrae como rechazo y superación permanente” (Rodríguez, 2007).

La Modernidad, entonces, es la emergencia de la razón como medida de toda las cosas, es desde sus comienzos un proceso emancipador de la sociedad, un salir de la tradición para adentrarnos en una secularización de las cosas, colocar al hombre como capaz de responder las interrogantes, colocando a la razón por encima de las respuestas teológicas, lo que en aquel tiempo era la tradición. La Modernidad se rebela contra las funciones normalizadoras de la tradición; la modernidad vive de la experiencia de rebelarse contra todo lo que es normativo (Habermas, 1994: 90), En este mismo sentido ella continúa

“Aparecen las estructuras de la racionalidad cognitivo-instrumental, la moral-práctica y la estético-expresiva, cada una de ellas bajo el control de especialistas que parecen más expertos en ser lógicos de estas particulares maneras que el resto de la gente” (1994: 94).

Hasta aquí no encontramos diferencias relevantes con la visión de modernidad de Dussel, ya que, en ambos puntos de vista, la modernidad es vista como un proceso de secularización en donde la razón instrumental se emancipa y se coloca como discurso hegemónico, dejando así en una posición secundaria tanto a la escolástica como el buscar respuestas desde la teología; otra faceta de la modernidad es que el hombre está en constante lucha con la naturaleza, queriendo dominarla y dejarla bajo sus pies.

"La Modernidad, más allá de la heterogeneidad de sus contenidos, se percibe a la manera de un conjunto coherente de racionalidad y progreso ético-social, cuyo debilitamiento es sentido por muchos en forma de verdadera amenaza [...] el conocimiento corresponde a un 
modelo objetivo y científico, validado por la experiencia y el progresivo dominio de la naturaleza, consolidado en un desarrollo de la técnica. Ello confluye en una superior emancipación del individuo y en el logro de mayores cotas de libertad y justicia social como horizonte paulatinamente alcanzable. Es esta Utopía la que cohesiona un modelo, cuya quiebra, desde su propio punto de vista, no puede sino conducir a la barbarie” (Rodríguez, 2007).

La Modernidad, es vista así como un proyecto de la ilustración europea, es decir que se establece como tal con la Revolución Francesa y con la revolución industrial, traspasando el siglo XIX hasta llegar aproximadamente a la década de 1950 y comienzos del 60’ ; modernidad que se vuelve mundial, hegemónica y con carácter totalizador para el resto del mundo.

Para Rodríguez Magda, a la sociedad industrial corresponde la cultura moderna, caracterizada por tener el patrimonio de la realidad, aspirando a su transformación, se halla indisolublemente unida a la noción de tiempo por su propio talante de innovación y progreso; una temporalidad histórica que busca de forma ilustrada se dirige hacia lo mejor, o, tal como dice la autora, en términos hegelianos “el cumplimiento del Espíritu Absoluto”. La industrialización, el maquinismo, las revoluciones, las utopías sociales pretenden realizar un avance histórico progresivo (Rodríguez, 2007). Dicho de otra forma, se consideran ser eje central de la cultura europea como fórmula para medir el "grado cultural o civilizatorio" del resto del mundo. Se toma a Europa como el fin al cual debe aspirar toda nación y cultura. A lo que pretende llegar esta concepción es tener un concepto totalizador de cultura, concibiendo todo lo no-europeo como barbarie. La modernidad es así, la visión de futuro, del progreso, del discurso unilateral que invita a las demás culturas a seguir el proyecto europeo: el "tiempo de las teorías fuertes”. Tenemos en vista una Modernidad que se autosustenta como proyecto europeo desde Europa, sin considerar al resto del mundo como parte constitutiva de aquel fenómeno, ç lo que lo lleva a un ensimismamiento y, por tanto a una visión bastante estrecha sobre el desenvolvimiento del proceso de nacimiento y desarrollo de la Modernidad, fenómeno que al contrario de lo que pretende, recoge múltiples componentes del sistema-mundo para poder establecerse, independientemente de ser el discurso Europeo por excelencia; la Modernidad es construida con la colaboración de otras culturas y territorios, cosa que Dussel toma en cuenta para el análisis de este fenómeno.

\section{b. La modernidad desde Enrique Dussel}

El mirar la Modernidad desde esta perspectiva, nos remite a una fecha anterior a la establecida por el discurso más tradicional quew establece el nacimiento de este fenómeno con la Revolución Francesa y la Revolución Industrial. Por el contrario, Dussel nos propone la fecha 1492 como inicio de la Modernidad, de una primera Modernidad; y sitúa los dos hitos anteriormente mencionados como parte de una segunda Modernidad, primera diferencia entre ambos autores. Para Dussel, la Modernidad se origi- 
nó en las ciudades europeas medievales, libres, centros de enorme creatividad (1992: 10); y el autor nos dice que en el momento en que Europa puede confrontarse con el otro,la modernidad emerge; es decir, cuando pudo definirse como un "ego" descubridor, conquistador, colonizador de la Alteridad constitutiva de la misma Modernidad (1992). Esta definición emana de una visión de la Modernidad desde "fuera de ella", desde fuera del proyecto europeo, capaz de reconocer la contrapartida de lo que se entiende por este fenómeno.

Enrique Dussel nos propone dos conceptos de modernidad:

El primero es eurocéntrico, provinciano, regional. La Modernidad es una emancipación, una "salida" de la inmadurez por un esfuerzo de la razón como proceso crítico, que abre a la humanidad a un nuevo desarrollo del ser humano. Este proceso se cumpliría en Europa, esencialmente en el siglo XVIII (2000: 45).

Esta visión es denominada eurocéntrica porque coloca como punto de partida de la Modernidad hechos y fenómenos intra-europeos, y el desarrollo posterior no necesita más que Europa para explicar el proceso (Dussel, 2000). En esta primera visión de la Modernidad es donde pretendemos colocar a Rodríguez Magda, ya que ella sólo se remite a Europa para construir su concepción de este fenómeno. La segunda visión que nos propone Dussel, es una visión que él cataloga mundial:

[...] la modernidad, en un sentido mundial [...] consistiría en definir como determinación fundamental del mundo moderno ${ }^{1}$ el hecho de ser (sus Estados, ejércitos, economía, filosofía, etc.) “centro” de la Historia Mundial. Es decir, nunca hubo empíricamente Historia Mundial hasta el 1492 (2000: 46).

Lo que nos plantea el autor, es que gracias a la invasión europea a América, occidente fue capaz de erigirse como el centro de la Historia, ya que la llegada de Portugal al Extremo Oriente en el siglo XVI como de España a América, la historia del planeta se torna una sola Historia Mundial, en otras palabras, gracias a estos hechos se arma lo que Wallerstein llamaría el sistema-mundo.

Otro aspecto importante, es que en consecuencia la colonialidad es constituyente de la Modernidad y podemos decir que para este autor este hecho es el sustento que hace posible emprender el proyecto europeo:

La primera etapa "Moderna": el mercantilismo mundial. Las minas de plata de Potosí y Zacatecas (descubiertas en 1545-1546) permiten acumular riqueza monetaria suficiente para vencer a los Turcos en Lepanto veinticinco años después de dicho hallazgo (1571). El Atlántico suplanta al Mediterráneo [... ] El siglo XVII (p.e. Descartes, etc.) son ya el fruto de un siglo y medio de "Modernidad": son efecto y no punto de partida (Dussel, 2000: 46). 
Otra cita que nos refuerza y aclara la idea es la siguiente:

Esa "periferia” de la Modernidad es su "otra-cara” (como la "otra cara" de la moneda o de la luna, que no se ven) constitutiva. No hay Modernidad sin “modernizado”, civilización sin “bárbaro” (Dussel, 2007).

Entonces, la industrialización como también La Ilustración. vendría siendo para Dussel la segunda etapa de la Modernidad. Ambas son la profundización de un proceso que comienza en el siglo XV. En este proceso Inglaterra reemplaza a España como potencia hegemónica, considerando que para el autor, España es el primer Estado-Nación moderno .

La configuración de la modernidad en Europa y de la colonialidad en el resto del mundo (con excepciones, por cierto, como el caso de Irlanda), fue la imagen hegemónica sustentada en la colonialidad de poder que hace difícil pensar que no puede haber modernidad sin colonialidad; que la colonialidad es constitutiva de la modernidad, y no derivativa (Mignolo, 2000: 61).

Entonces, la visión de Modernidad para Enrique Dussel y con él para todos los autores postcoloniales se forja desde la concepción de una visión mundial, esto quiere decir, articulando fenómenos intra-europeos con fenómenos ocurridos en las demás áreas del planeta, lo cual conforma el sistema-mundo que emerge en los siglos XV-XVI con Portugal y España como protagonistas. Por un lado tenemos la conexión con el Extremo Oriente y por el otro la llegada de los europeos a tierras americanas por parte de España. Posterior a esto, al situarse Inglaterra como imperio hegemónico, nos abre paso a la segunda Modernidad que conlleva el proceso de la industrialización.

\section{c. La Postmodernidad}

Este fenómeno según la propuesta de Rodríguez Magda, tiene lugar en la tríada Modernidad, Postmodernidad, Transmodernidad, aquella sería, en palabras de la autora, la tríada dialéctica que, más o menos,en términos hegelianos completaría un proceso de tesis, antítesis y síntesis (2007). Queremos decir con esto, que antes de abocarnos al plenamente al concepto de la Transmodernidad es necesario esbozar lo que significa la Postmodernidad para Rodríguez Magda, ya que esta es condición sine qua non para entender la Transmodernidad como continuidad y superación de este concepto.

La crisis de la Modernidad, el fin de la pujanza de las Teorías fuertes, nos aboca a un vacío epistemológico, a un hueco de legitimación. Quizás el peor corolario de las corrientes postmodernas haya sido la zambullida en el nihilismo, en el relativismo, en la banalización, en el eclecticismo del "todo vale” (Rodríguez, 1997: 13). 
La Modernidad se ancla, por tanto, en la posibilidad y legitimidad de los discursos globales. La crisis postmoderna atenta precisamente contra esta posibilidad y legitimidad (Rodríguez, 2007). Es la puerta a la atomización de los discursos, a la caída de los grandes relatos y con ello a la crítica al proyecto Moderno como vía del progreso, ya que de aquellos metarrelatos (Lyotard) se pasa a discursos contextualizados y situados; con lo cual se pone en duda la razón instrumental como medida de las cosas.

La sociedad moderna era conquistadora, creía en el futuro de la ciencia y la técnica; en la sociedad postmoderna se disuelven la confianza y la fe en el futuro, ya nadie cree en el porvenir de la revolución y el progreso, la gente desea vivir el "aquí" y "ahora”, buscando la calidad de la vida, y la cultura personalizada (Picó, 1994: 37).

Otra característica de los tiempos postmodernos es la emergencia de un individualismo hedonista por sobre un individualismo competitivo, que sólo tiene ojos para sí mismo y para su grupo. A nivel discursivo significa una deslegitimización de la modernidad europea, un quiebre. En palabras de Wellmer, la modernidad que acepta la pérdida de sentido, de valores y de realidad con una jovial osadía es el postmodernismo como "gaya ciencia” (1994). Para tener una definición un poco más acabada sobre lo que significa la postmodernidad, dentro de los márgenes de consenso existente, podemos establecer que:

Se está refiriendo a las condiciones culturales que se han dado a nivel planetario en las últimas décadas, caracterizadas por la producción de una nueva modalidad de subjetividad. Esta subjetividad es oscilante, variable, sin centro fijo ni continuidad de sentido y convicciones. De tal modo, da lugar a un comportamiento que se ha descrito como Light, poco comprometido con los otros y con las grandes causas éticas y políticas de interés colectivo o universal (Follari, 2005: 805).

Para Rodríguez Magda, la era postindustrial forma parte de la cultura postmoderna, una cultura que vaticina el fin de la historia y de los grandes relatos. Sin embargo, aquellos fragmentos diversos que son parte de esta dominante cultural, confluyen unos con otros, englobándose bajo un solo paradigma que la autora entiende como el resurgir de un Gran Relato, es decir el umbral de la Globalización.. Entonces, en este nuevo paradigma la Postmodernidad se va desvaneciendo para constituir la base de una nueva dominante cultural que ya no puede denominarse "post". Es por ello que Rodríguez Magda nos sugiere cambiar de prefijo, para denominarlo "trans”, superando así a lo "post”. Por lo tanto, sugiería tanto continuidad como ruptura con la Postmodernidad. Entramos así a la Transmodernidad.

A continuación analizaremos este concepto ya que si bien para Rodríguez Magda la Transmodernidad corresponde a una tríada (Modernidad, Postmodernidad, Transmodernidad), Dussel, plantea y desarrolla algo 
muy diferente, contrapuesto a la visión de esta autora. Dussel propone una visión pluricentrista como categoría de un discurso anti-hegemónico para establecer un diálogo intercultural, y no un sistema hegemónico que pretende ser totalizante, tal como lo reconoce Rodríguez Magda.

\section{d. La Transmodernidad desde Rosa María Rodríguez Magda}

La Transmodernidad(i), como se ha dicho, corresponde a una nueva etapa, a un nuevo reordenamiento cultural, a una superación de la Postmodernidad y con ello a una recuperación de algunos aspectos que quedaron inconclusos en la Modernidad. La Transmodernidad es la vuelta a un metarrelato: la globalización. Este fenómeno, para ella es considerado como el gran referente que marca la ruptura con la Postmodernidad y da paso a un nuevo fenómeno totalizante y totalizado ya que todo lo fragmentado, la atomización de relatos contextualizados son envueltos bajo un mismo manto, englobados bajo la globalización. La autora considera que la Transmodernidad es el mundo de lo virtual, de la ficción, una copia que suplanta al modelo. Es un intento de abrirse paso frente a los desafíos de nuestra época sin renunciar a los proyectos ilustrados (León, 2006). En fin, lo que engloba el término Transmodernidad es un intento por definir la realidad de hoy en día, tomando en cuenta que lo Post es un fenómeno anterior.

Suponemos que lo postmodernos ha acabado ya, aunque las grandes editoriales siguen publicando libros sobre ella. Fue un fenómeno que, aun comenzando en Estados Unidos a finales de los cincuenta con la poesía de los Black Mountain Poets y continuando en los sesenta con la literatura exhausta, en los setenta con la arquitectura, etc., alcanza sin duda su cenit en los años ochenta, que es cuando mejor se descubren los efectos de una corriente que acabó siendo una moda más del mercado (Rodríguez, 1998: 8).

Con la afirmación que la Transmodernidad es el fenómeno de nuestra época, Rodríguez Magda se refiere al retorno de algunas aspectos de la Modernidad, los más universales de ésta en términos de una copia, el retorno de una Modernidad débil, rebajada, light; esa es nuestra realidad: donde la copia suplanta al modelo. La Transmodernidad vendría siendo el Postmodernismo sin su inocente rupturismo, la galería museística de la razón, para no olvidar la historia, que ha fenecido, para no concluir en el bárbaro asilvestramiento cibernético o más-mediático. Es decir, es proponer valores como frenos o como fábulas (Rodríguez, 2007). Otra de las características de la Transmodernidad es que retoma y recupera los valores vanguardistas, los copia y los vende, pero a la vez recuerda que el arte ha tenido también un efecto de denuncia y que no todo vale; anula la distancia entre el elitismo y la cultura de masas (2007). Lo que se dice es que la Transmodernidad no es un deseo o una meta, es algo que simplemente está, y que la autora considera como un estado de la cultura, que, a la vez, significa una explosión variopinta de ideas que desparrama ideas en múltiples direcciones. 
Otro aspecto de la Transmodernidad, para XX, es el abandono de la representación, es el reino de la simulación, de la simulación que se sabe real (2007). En otras palabras,

La Transmodernidad, como etapa abierta y designación de nuestro presente, intenta, más allá de una denominación aleatoria, recoger en su mismo concepto la herencia de los retos abiertos de la Modernidad tras la quiebra del proyecto ilustrado. No renunciar hoy a la Teoría, a la Historia, a la Justicia social, y a la autonomía del Sujeto, asumiendo las críticas postmodernas, significa delimitar un horizonte posible de reflexión que escape del Nihilismo, sin comprometerse con proyectos caducos pero sin olvidarlos (Rodríguez, 2007).

Característico de esta época es también la hegemonía de la ausencia, la carencia de los fundamentalismos y la nostalgia de ellos como la nostalgia del todo y del fundamento.

Los elementos fundamentales de la Transmodernidad de Rodríguez Magda son: la globalización como hecho social; la Transmodernidad como paradigma para desafiar los intentos de un proyecto ilustrado contemporáneo; y la utilización del enfoque dialéctico como generador de cambio social. Realiza un análisis del concepto desde la óptica gnoseológica, metafísica, ético política, la subjetividad, lo sacro y lo estético.

Para Enrique Dussel. Esta concepción conlleva serios conflictos a causa de su etnocentrismo y por lo tanto de exclusión de una gran mayoría.

\section{e. La Transmodernidad de Enrique Dussel}

La Transmodernidad(ii) no es un estado de la cultura actual, no es una continuidad de la postmodernidad ni parte de la tríada dialéctica de la que habla Rodríguez Magda. Tampoco es una especie de radiografía cultural ni un término rabiosamente último como lo expresa la autora. La Transmodernidad es un proyecto que va por fuera de la Modernidad y de la Postmodernidad, es un proyecto paralelo que surgiría fuera de Europa y de Estados Unidos, oponiendose así al carácter totalizante que tiene el proyecto moderno europeo. A diferencia de Rodríguez Magda, Dussel realiza un planteamiento mucho más historicista del término, y a juicio nuestro, mucho más cercano a nuestra realidad, ya que la idea transmoderna significa ver y analizar los fenómenos de manera mundial, desde el sistema-mundo, y no solamente desde categorías intraeuropeas y eurocéntricas.

Hablar en cambio de "Trans»-modernidad exigirá una nueva interpretación de todo el fenómeno de la Modernidad, para poder contar con momentos que nunca estuvieron incorporados a la Modernidad europea, y que subsumiendo lo mejor de la Modernidad europea y norteamericana que se globaliza, afirmará «desde-fuera” de ella componentes esenciales de sus propias culturas excluidas, para desarrollar una nueva civilización futura, la del siglo XXI (Dussel, 2001: 390). 
Lo que hay detrás de la posición de Dussel es un esfuerzo por interpretar de manera no eurocéntrica la historia, y plantear un proyecto a futuro, utópico, un planteamiento de diálogos interculturales simétricos. La idea de Transmodernidad lleva consigo el propósito de resurgir lo propio de cada cultura, aquello que dejó de lado el proyecto moderno por ser inconmensurablemente disímil a su propia ideología.

Europa comenzó a ser "centro" del mercado mundial (y extender por ello el sistema-mundo a todo el planeta) desde la revolución industrial; que en el plano cultural produce el fenómenos de la ilustración, cuyo origen, in the long run, debemos ir a buscarlo [...] en la filosofía averroísta del califato de Córdoba. La hegemonía central e ilustrada de Europa no tiene sino dos siglos (1789-1989). ¡Sólo dos siglos! Demasiado corto plazo para poder transformar en profundidad el "núcleo ético-mítico" (para expresarnos como Ricoeur) de culturas universales y milenarias como la china y otras del extremo oriente (como la japonesa, coreana, vietnamita, etc.), la indostánica, la islámica, la bizantino-rusa, y aún la bantú o la latinoamericana (de diferente composición e integración estructural) (Dussel, 2005).

La Transmodernidad se propone ver desde el otro los fenómenos y discursos hegemónicos que se producen y se enuncian desde el centro.

Desde la cultura europea-estadounidense, las otras culturas que son asimétricas tanto en el aspecto económico, militar, político, científico como tecnológico, guardan en sí una alteridad con respecto a la Modernidad europea, con la cual se han interrelacionado y convivido, y han aprendido, a la vez, a responder a su manera a los desafíos de ésta (Dussel, 2005). El autor nos dice que estas culturas sólo están en un proceso de letargo, y que en la actualidad comenzarían a despertar; estando en un proceso de pleno renacimiento buscando caminos nuevos para su próximo futuro. A diferencia de Rodríguez Magda, Dussel considera que las culturas exteriores a la occidental, por no ser modernas tampoco pueden caer en la categoría de postmodernas. Son premodernas (más antiguas que la modernidad), conviven con ella y a futuro serán transmodernas. Dussel nos dirá entonces que las culturas, por ejemplo la china (en la cual centra principalmente sus ejemplos), no podrán caer nunca en las categorías del postmodernismo occidental, Al contrario, partiendo desde sus propias raíces, tendrán su desenvolvimiento propio La Transmodernidad es, entonces, un proyecto mundial, historicista y cultural que mira desde un "otro", capaz de tener voz ante el discurso hegemónico occidental. La Transmodernidad llega así a significar una co-realización de lo imposible para la sola Modernidad; es decir un diálogo entre culturas.

Así el concepto estricto de "trans-moderno" quiere indicar esa radical novedad que significa la irrupción, como desde la Nada, desde Exterioridad alternativa de lo siempre Distinto, de culturas universales en proceso de desarrollo, que asumen los desafíos de la Mo- 
dernidad, y aún de la Post-modernidad europeo-norteamericana, pero que responden desde otro lugar, other location. Desde el lugar de sus propias experiencias culturales, distinta a la europeo-norteamericana, y por ello con capacidad de responder con soluciones absolutamente imposibles para la sola cultura moderna² (Dussel, 2005).

Transmodernidad es entonces todos los aspectos que están más allá, y más acá, de las estructuras valoradas por la cultura moderna europeonorteamericana. Estos aspectos siguen vigentes en las culturas universales no-europeas, y que el autor establece que se han puesto en dinamismo hacia una utopía heteroglósica, de diálogos simétricos, no solo de apariencia como ocurre en la modernidad, sino de simetría real, y de he ahí el carácter utópico y futuro. Es un intento de quiebre del eurocentrismo y, por lo tanto, de la propuesta transmoderna de Rodríguez Magda.

\section{Palabras finales}

Rodríguez Magda discrepa claramente de Dussel en su concepto de Transmodernidad reconociendo que su propia propuesta teórica corresponde a una concepción del primer mundo, de ese mundo globalizado y que ella lo caracteriza como vacío, estableciendo además que:

La Transmodernidad no es la panacea a todas las contradicciones, y caeríamos en la impostura intelectual si, guiados por la magia del nombre, pretendiéramos fabricar la transmodernidad de los pobres, la transmodernidad de los bárbaros, la transmodernidad de los iluminados. Buscar la cuadratura del círculo en clave de un pensamiento fuerte multicultural es prolongar la lógica de la modernidad, sin comprender que nos hallamos ya muy lejos de ella. Pensar con la nueva lógica es deshacernos de una vez por todas de las antiguas falsas ilusiones (Rodríguez, 2007).

La autora nos remarca que la Transmodernidad no pretende resolver nada, solo es el nuevo paradigma del primer mundo, sofisticado. Y por eso es muy distinto pretender "angelizar" al excluido o al fundamentalista, vendiendo como anheladas síntesis lo que no son sino beatíficas "buenas intenciones" de la mano de los teólogos de la liberación, el mesianismo New Age o la jerga políticamente correcta de los organismos internacionales (2007). También Rodríguez Magda nos dice que la Transmodernidad no es una ONG para el tercer mundo, que es simplemente el lugar donde están “ellos", los del primer mundo. Digamos, entonces, que es precisamente donde no están los excluidos.

Lo que nos dice Dussel, es muy diferente. Sin embargo, este autor no hace alusión a Rodríguez Magda, ni siquiera referencias subrepticias; más bien se refiere a los filósofos mayores de Europa como Habermas y Vattimo. Sin duda aquí se encuentra también Rodríguez Magda como filósofa que toma las ideas de estos pensadores. Dussel propone un claro proyecto 
de quiebre con el eurocentrismo y el discurso totalizante de la Modernidad/ Postmodernidad, y nos plantea una visión desde el excluido. Dussel nos dice que el punto de partida al que se refiere tiene tras sí un hecho con relevancia ética: en cifras, el 20\% más rico de la tierra consume-destruye el $82.7 \%$ de los bienes del planeta, mientras el 20\% más pobre consume sólo el 1.4\% de dichos bienes (2007). Se puede estar con la modernidad en la periferia, pero la Transmodernidad es un proyecto nuevo de liberación de las víctimas de la Modernidad, la idea es entrar en diálogo, pero sobretodo tener el espacio para desarrollar las potencialidades alternativas de cada cultura. El proyecto transmoderno de Enrique Dussel se desprende de su filosofía, ética y política de la liberación, que tiene un carácter de denuncia y ruptura con el eurocentrismo que es hegemónico en los discursos institucionalizados. 


\section{Notas}

${ }^{1}$ Cursivas del autor.

${ }^{2}$ Cursivas del autor.

(i) El concepto Transmodernidad es utilizado primeramente por Rodríguez Magda, la primera aparición del concepto es en 1989, cosa que ella remarca en sus trabajos referidos al tema

(ii) Aquella propuesta mundial Dussel la viene trabajando desde hace mucho tiempo, 1965 aproximadamente, pero el proyecto transmoderno, refiriéndose al concepto propiamente tal, es de la década de los $90^{\prime}$. 


\section{Bibliografía}

Bautista, Juan (2005), “¿Qué significa pensar desde América Latina?”, en Revista Comunicologí@: indicios y conjeturas, publicación electrónica del departamento de comunicación de la Universidad Iberoamericana. México, primera época, $\mathrm{N}^{\circ} 4$, otoño, disponible en: http:// :revistacomunicologia.org/index.php?option=com:_content\&task =view\&id=118\&itemid=89

Dussel, Enrique (1992), 1492: el encubrimiento del otro: hacia el origen del mito de la modernidad. Editorial Nueva Utopía, Madrid.

Ídem (1998), Ética de la Liberación en la edad de la globalización y de la exclusión. Editorial Trotta, Madrid.

Ídem (2000), “Europa, modernidad y eurocentrismo”, en Lander, Edgardo (Comp.), La colonialidad del saber: eurocentrismo y ciencias sociales: perspectivas latinoamericanas. CLACSO, Buenos Aires.

Ídem (2001), Hacia una política crítica. Desclée de Brouwer, Bilbao.

Ídem (2004), “Sistema-mundo y Transmodernidad”, en Banerje, Ishita; Dube, Saurabh; Mignolo, Walter (eds.), Modernidades coloniales. Editorial El Colegio de México, México.

Ídem (2005), “Transmodernidad e Interculturalidad (interpretaciones desde la Filosofía de la Liberación), disponible en: http://www.afyl.org/ transmodernidadeinterculturalidad.pdf

Ídem (2007), “Un diálogo con Gianni Vattimo: de la Postmodernidad a la Transmodernidad”, en Revista filosófica A Parte Rei, $N^{\circ} 54$; disponible en: http://serbal.pntic.mec.es/AParteRei

Follari, Roberto (2005), “Postmodernidad”, en Salas, Ricardo (coord.), Pensamiento Crítico Latinoamericano: conceptos fundamentales. Editorial Universidad Católica Silva Henríquez, Santiago, Chile.

Habermas, Jürgen (1994), “Modernidad versus postmodernidad”, en Picó, Josep (comp.), Modernidad y postmodernidad. Editorial Alianza, Madrid.

Jameson, Fredric (2001), Teoría de la posmodernidad. Editorial Trotta, Madrid.

Lyotard, Jean-François (1991), La condición postmoderna. Editorial Red Editorial Iberoamericana, Buenos Aires.

Mignolo, Walter (2000), “La colonialidad a lo largo y a lo ancho: el hemisferio occidental en el horizonte colonial de la modernidad”, en Lander, Edgardo (comp.), La colonialidad del saber: eurocentrismo y ciencias so- 
ciales: perspectivas latinoamericanas. CLACSO, Buenos Aires.

Picó, Josep (1994), Modernidad y postmodernidad [introducción]. Editorial Alianza, Madrid.

Rodríguez, Rosa (1997), El modelo Frankenstein: de la diferencia a la cultura post. Editorial Tecnos, Madrid.

Ídem (1998), Y después del postmodernismo ¿qué? Editorial Anthropos, Barcelona.

Ídem (2007), “Transmodernidad; La globalización como totalidad transmoderna”, en Revista Observaciones Filosóficas, $N^{\circ} 4$; disponible en: http://www.observacionesfilosoficas.net/latransmodernidadlaglo.html

León, Luz (2006), “¿Nueva épica del sentido en la Transmodernidad? [crítica del libro Transmodernidad de Rosa Rodríguez]”, en Revista Internacional de Filosofía y Política, Julio, No 27, Madrid; disponible en: http:// e-spacio.uned.es:8080/fedora/get/bibliuned:filopoli-2006-27-28E3A715DB55-9D36-FCE0-67AD2C74F9BB/nueva_epica.pdf

Wellmer, Albrecht (1994), “La dialéctica de la modernidad y postmodernidad”, en Picó, Josep (comp.), Modernidad y postmodernidad. Editorial Alianza, Madrid.

Recibido: 20.02.2012

Aceptado: 09.10.2012 